\title{
ACTIONABLE FRAMEWORK FOR CITY DIGITAL TWIN-ENABLED PREDICTIVE MAINTENANCE AND SECURITY MANAGEMENT SYSTEMS
}

\author{
SOFIA AGOSTINELLI \\ Department of Astronautics, Electrical and Energy Engineering, Sapienza University of Rome, Italy
}

\begin{abstract}
The growing potential offered by the use of ICT (Information Communication Technology)-based approaches for process management in the built environment is increasingly used to configure effective digital twins of products and processes. Focusing on Smart Cities, the realization of integrated threedimensional BIM (Building Information Modeling) and GIS (Geographic Information Systems) provides microscopic and macroscopic geometric databases containing static, dynamic, geometric and semantic data representing the information centre for an efficient management of the lifecycle in vertical and horizontal systems. Therefore, processing data and information about the lifecycle of city assets through a Digital Twin Model ensures considerable support to the process management of built environments. Through the integration of these models with Artificial Intelligence (AI) systems it becomes possible to increase the optimization and progressive functional automation of the activities interconnected to the assets' lifecycle. The proposed research presents a full-digital actionable framework based on pervasive and ubiquitous systems for security and facility management based on City Digital Twins. An integrated digital system for security management is introduced through the use of self-learning systems for unmanned security based on image recognition and AI. Finally, even maintenance operations can benefit from the configuration of predictive maintenance systems aimed at reducing costs and failures through a digital information management system combined with analytics based on data flows from sensors and historical data.
\end{abstract}

Keywords: digital twin, BIM, artificial intelligence, machine learning, security management, predictive maintenance, facility management, smart cities.

\section{INTRODUCTION}

Modern challenges proposed by the increasingly digitization of industrial processes, give the opportunities of developing new digital scenarios and management tools, which combined with the potential of Artificial Intelligence (AI) and Machine Learning (ML) systems, allows to optimize and automate the construction process through Cyber-Physical systems (CPS).

CPS can be defined as systems of systems, composed of heterogeneous, collaborating and ubiquitous components both physical and virtual as a Digital Twin (DT) of reality [1].

Defining a Digital Management Framework is the key objective for an effective fulldigital building process and ecosystem based on three-dimensional information models, replicating physical objects such as buildings, managing and monitoring their interactions with reality.

The growing potential offered by ICT (Information Communication Technology)-based approaches opens renewed scenarios for process management in the built environment through BIM (Building Information Modeling) and GIS (Geographic Information Systems) integrated approaches. In fact, it becomes possible to microscopic and macroscopic geometric databases containing static, dynamic, geometric and semantic data representing the core information for an optimized management phase in the life cycle of vertical and horizontal systems. Through the integration of BIM models with AI systems it is possible to increase the optimization and progressive functional automation of the activities interconnected to the life cycle of assets, configuring Digital Twin (DT) systems. 
DT is a concept deriving from the high-value manufacturing industries, and it is going to be gradually used also in the construction sector. DT of buildings reproduce their geometric and semantic information as 3D models configured and structured according to databases criteria, where data are contained within objects composed of specific parameters and attributes describing the components themselves, thus enclosing useful information for the definition and simulation of processes.

The DT system is able to improve and enriching its knowledge and data receiving input and signals from sensors, constantly monitoring buildings, developing self-learning capabilities and predictivity through the integration with AI systems.

Therefore, the concept of DT is extremely transversal and applicable to various and diversified areas.

The so-called Key Enabling Technologies allow the configuration of Digital Twins combining sensors for detecting and transmitting data, computing power for data processing, and the hardware and software for analyzing and simulating specific scenarios. This framework allows to configure systems for managing urban metabolism, as well as configuring predictive maintenance of building systems defining intelligent human-scaled urban environments.

From literature analysis different applications of City Digital Twins emerged: the KIRAdigi pilot project is aimed at producing the DT of the Kalasatama area near to Helsinki [2], as a smart city development configuring a platform for designing, testing, applying and servicing the entire lifecycle of the built environment; Virtual Singapore is a dynamic threedimensional city information model and collaborative data platform of Singapore [3], aimed at public, private and research uses enabling users to join simulation, planning and decisionmaking tools, solving emerging challenges for the city; even the DT of the port of Rotterdam [4], using AI analyzing data, is able to do accurate real-time predictions about moor and departures, reducing waiting times and costs.

As described in Fig. 1, the present paper identifies specific experimental domains for City Digital Twin such as security management, i.e., the physical security of places through advanced computer vision-based surveillance cameras, and facility management, i.e., the management of the built environment through predictive maintenance.

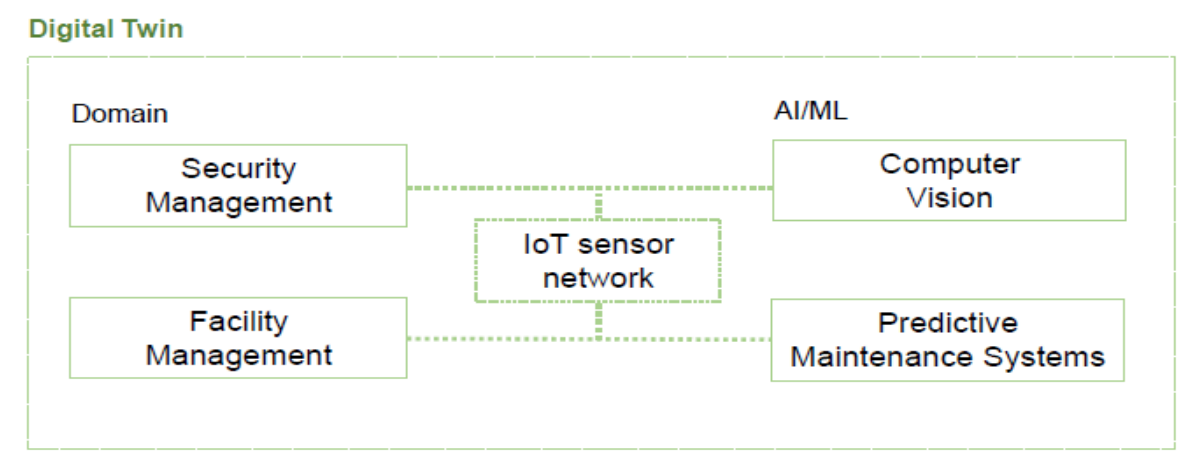

Figure 1: DT-based domain and technology integration.

\section{CITY DIGITAL TWIN ENABLING TECHNOLOGIES}

The smartification of digital systems towards defining Collaborative CPS as a combination of virtual and physical assets, has determined the need for specific digital design approaches. 
A framework has been developed achieving generic scenarios bridging them on promising real cases. Considering the integration of physical and virtual worlds as a significant support for people daily experiences, from business to healthcare or elderly care. An integration of data flows coming from different sources as well as predictive models based on historical data and current performances of the physical system is necessary to achieve effective DTs purposes.

Therefore, the development of a Digital Twin Model (DTM) is supported from the configuration of a multidisciplinary integrated information model (BIM), containing useful process information and communicating data coming from different sources. Moreover, AI provides DTs with learning capabilities, implementing autonomous actions based on the performed elaborations.

The configuration of autonomous systems is aimed at analysing and processing data, implementing corrective actions, reducing errors, waste and systems malfunctions.

The described construction process of the DT at macroscopic level meets the synergy between Big Data, i.e. a huge amount of data about the city and AI systems, capable of processing and analysing them to implement strategies. Therefore, the very first DT configuration phase is related to the production of structured data and information, coming from their collection in databases and information models, up to data processing and analysis.

Thus, configuring the DT of a residential district can be a key tool for the storage, visualization, analysis and creation of data useful for the management of urban life. Moreover, given the absolute centrality of data systems in the configuration of digital processes, the integrated use of GIS and BIM technologies allows information management both at the building and territorial scale.

Therefore, although they share the essential concept of describing the real world through the combination of visual representations and information, BIM and GIS are conceived and developed as belonging to different domains. In fact, they present divergences in the level of detail, as BIM can be used to create, manage and share lifecycle data of vertical structures, e.g. buildings, while GIS can store, manage and analyze data describing the horizontally distributed urban environment.

\subsection{Internet of things for smart cities}

The Internet of Things (IoT) allows connections between physical world and virtual information at any time, in any place and between anything [5], defining the configuration of smart systems through heterogeneous and ubiquitous objects [6] originating the development of interactive object automation to exchange information [7].

However, one of the main components of IoT systems are the Wireless Sensor Networks (WSN) [8] which interconnect sensors in order to obtain data stored in a server to analyze and automate tasks. Actuators allow the execution of actions on motors, machines, and so on. Moreover, WSN and actuator systems can be connected in systems known as Wireless Sensor and Actuator Network (WSAN). Also, Smart Objects are considered as core IoT components, as they can perform actions having sensors capabilities and being able to process information as well as performing actions using an internet connection.

In the configuration of smart urban systems and Smart Cities, different types of sensors can be distributed to gather information about city management processes. The integration of ubiquitous communication networks, WSN, WSAN, and intelligent systems, allow Urban Intelligence, improving citizens daily life [9]. Some European Smart Cities like Luxemburg defined the Smart City concept basing on six indicators and a European project called 
SmartSantander is another example [10], proposing an IoT architecture for Smart Cities, showing and offering different urban services.

\subsection{Computer vision for enhanced security management}

Modern security management systems for buildings, residential complexes and urban areas are increasingly based on technological "unmanned" systems, capable of acquiring, interpreting and finally suggesting methods to ensure the safety of the inhabitants.

Being able to have a digital model allows security managers to virtually test the effectiveness of the positioning of control IoT sensors, as well as the surveillance systems, and the timeliness of the implementation of consequent actions to secure the monitored scene. Current security systems are based on video cameras, drones and perimeter and volumetric motion IoT sensors, but the use of image recognition algorithms allows to detect potentially dangerous situations, without the human presence in operating rooms.

AI applications based on self-learning capabilities can benefit from a DT system that allows to enrich the database with the results of simulations aimed at obtaining a virtual replica of the controlled area.

Computer vision is an emerging approach to advanced digital security management using facial recognition applications. The use of computer vision as a public safety tool is still under exploration, focusing on the possible integration of advanced surveillance cameras computers for a possible inexpensive solution for digital security management. Computer vision offers the main benefit of substituting human monitoring with automated analysis, where the human assumes a supervisory assessment and decision role.

Computer vision digitizes a visual image into a grid of pixels where a numerical value is assigned to each pixel, representing its colour. This process generates a two-dimensional grid of numbers for each video frame.

The assigned numbers are fundamental for enabling the computer vision analysis, interpretation and labelling capabilities, defining a picture-to-number process making computer vision possible.

In essence, computer vision processes determine a numerical amount of pixel values providing information about an image or frame, such as identifying people and places, face recognition and image stabilization for automated real-time video stream analysis or even post-hoc searches of archived video files.

Live real-time video analysis involves the possibility to promptly identify and correctly respond to ongoing events, as the capability and effectiveness of a surveillance system is in reducing crime through real-time intervention, also reducing the amount of time currently spent reviewing and searching video frames.

An online dictionary learning approach to detect abnormalities is being pursued to cope with these challenges, cutting long videos into small clips and comparing detected elements with those in a dictionary for the identification of abnormalities.

Computer vision capability is also to automatically analyze video frames and screen out irrelevant information [11]. An identified solution approach is to use semantic indexing in computer vision, deep learning and foreground object detection to determine a small set of real-time suspicious video clips from multiple camera feeds or from a video archive [12].

The proposed DT system is based on the configuration of an integrated digital system for unmanned security management using self-learning systems and AI based on image recognition, which increase security levels in urban areas, minimizing the risks of aggression, theft, intrusion and forcing, gatherings etc. [13]. 
Therefore, the objective is the configuration of a completely unmanned security system, through the integration of control systems with self-learning and AI based on image recognition.

This allows to increase the overall security levels by reducing the risk of attacks, thefts, intrusions and break-ins through specific algorithms capable of recognizing images through a network of video surveillance cameras integrated with AI systems.

Through the DTM it is possible to evaluate data coming from the risk database based on a BIM Model which contains a specific analysis of the areas subjected to computer vision, according to the risks associated to the current security conditions. The DTM also provides analysis and simulation for different design solutions suggesting camera types according to the lighting system necessary to ensure high levels of security.

Then the result of the DTM-based risk analysis is the definition of a level of danger for each area evaluated through a security system that can identify the nature of the area as well as its current level of security, assigning a tag for the digital monitoring of the entire area.

\subsection{Predictive maintenance systems}

Building Management systems based on predictive maintenance [14], [15] are going to radically change the way buildings and complex areas are going to be maintained. The DTM will help facility managers to easily access data and promptly carry out operations and maintenance works even remotely, having data available to ensure constant monitoring [16]. Consequently, the DTM can be used to evaluate the current condition of the asset [17]; to predict future behaviours, both in conditions of normal obsolescence or referring to extreme events, through the improvement of performance control [18], [19] and the possibility of constantly optimizing operations.

In fact, the added value of predictive maintenance [20] consists in the ability to determine the remaining useful life of a plant, as well as of a component or a material, to establish what will be the most appropriate time to perform maintenance or replacement of individual elements [21].

The DTM will allow facility managers to make predictions about what will happen to a building or infrastructure in the future [22], [23] creating new possibilities for optimized activity planning and scheduling, allowing maintenance managers to get real-time detailed reports about each individual system, accurately monitoring performances over time.

Thus, AI is the enabler of prediction according to DTM-based simulations [24] and analysis, depending on the amount of available data as time series [25].

In fact, before the advent of AI and DTMs, facility managers used to spend hours in evaluating costs and benefits of any intervention before implementing it, without having support from real data to evaluate and simulate the actual success chances and the consequences of their choices [26]. Otherwise, DTM-based predictive maintenance allows facility managers to test every single element before activating it, and to analyze in advance what will be the impact of each decision [27], promptly responding to any failures [28]. Thanks to the amount of data continuously collected through sensors, more detailed analysis can be performed highlighting new management possibilities [29].

\subsection{Digital twin-based advanced building management systems}

As an example, the operations and maintenance of a residential district can benefit from the configuration of predictive maintenance systems aimed at reducing operating costs and malfunctions [30], through the definition of a digital maintenance system based on IoT [31] 
and historical data, continuously updated through the DTM. Fig. 2 shows the DT framework architecture.

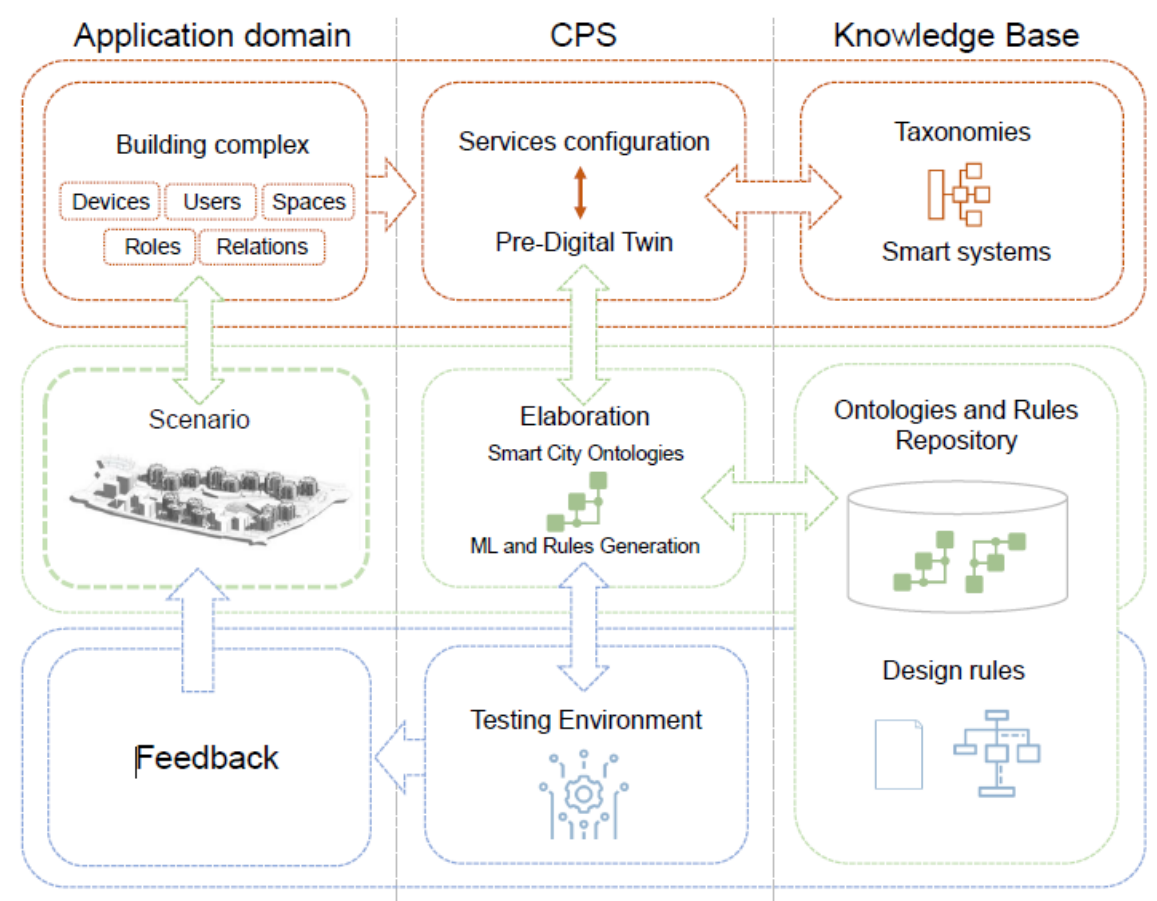

Figure 2: DT framework architecture.

Moreover, the techniques of Machine and Deep Learning combined with mechanisms of automatic reasoning allow to strategically exploit the large amount of information available, through features for (1) the acquisition of heterogeneous data streams; (2) prediction of failures; (3) profiling of users' behaviors and operating states of machinery; (4) optimized planning of raw material supplies and processing shifts; (5) real-time monitoring of systems and infrastructures; (6) multidimensional analysis of information.

The proposed solution is based on automatic reasoning, which is complementary to the inductive models, monitoring the scheduled maintenance activities using rule-based approaches for the definition of malfunctioning scenarios, aiming at a progressive decrease of breakdown interventions.

The BIM Model has a central role in the architecture of the information management system, as a physical and 3D spatial information container aimed at optimizing operations and maintenance interventions, integrating ML systems using rule-based approaches such as association rule mining [32].

Therefore, in the present framework it seems useful to find a hierarchy in the classification of spaces using clustering [33] machine learning techniques, which automatically identify groups/classes of similar spaces for their digital representation through a scalable and modular approach.

The implementation of the proposed system is based on the integration of three components: 
- data from BIM three-dimensional information containers;

- data flows automated through visual programming systems (e.g. Autodesk Dynamo) for a bidirectional connection of the WSN, controlled by AI systems, to the BIM Model, configuring a reliable DTM;

- ML systems for data processing using AI based on Phyton, due to efficiency, reliability and compatibility reasons with flows programmed through BIM-based visual programming systems; the AI system could be implemented through mixed techniques of hybrid reasoning and regression analysis [34].

Then, in the context described above, closed circuit television (CCTV) systems represent an additional source of data useful even for optimizing monitoring as well as for the identification of breakdowns, which can be associated and integrated with the alerts generated by Ticketing and Building Management System (BMS) as described in Fig. 3.

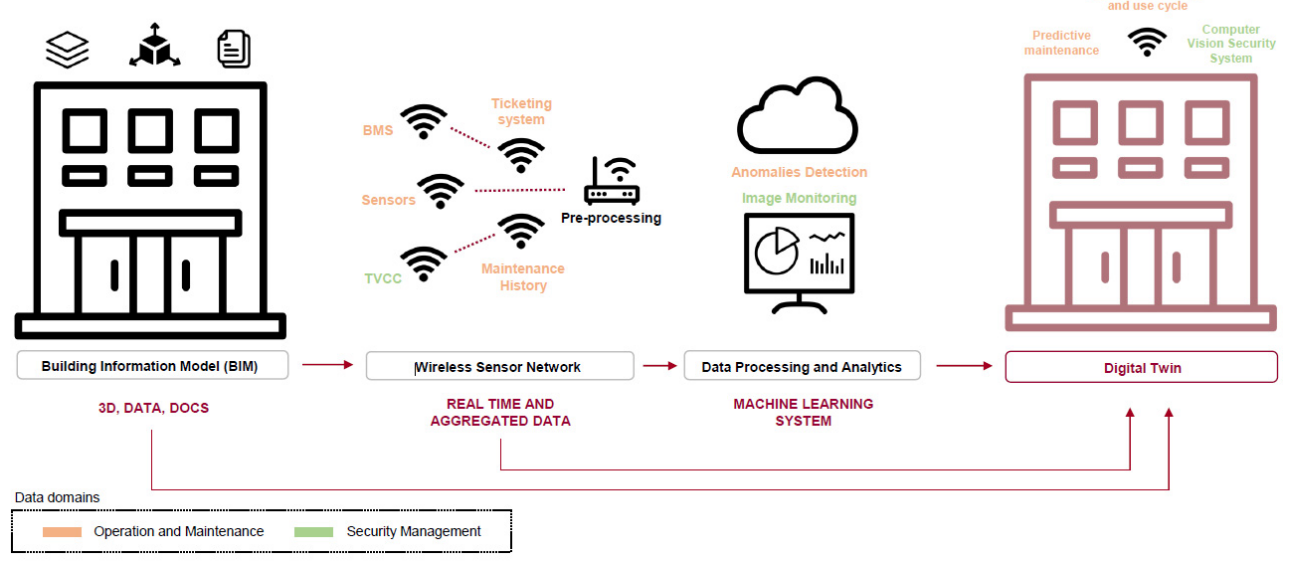

Figure 3: Functional system architecture.

In fact, cameras capture day and night images for security reasons even in closed or unreachable environments. Consequently, they can be considered as a source of useful data for machine learning and computer vision systems to process and compare the chromatic range variation [35] as the performance of the lighting fixtures decreases, or the state of conservation of elements/components that are difficult to reach (roofs, facade elements, etc.).

As described, the main objective is the reconfiguration of the operations and maintenance model traditionally limited to plant operation and fault interventions, by implementing digital and intelligent systems for predictive maintenance based on sensors and historical data [36].

As defined, the virtual monitoring through digital systems can reduce maintenance interventions allowing a considerable saving on the overall costs, eliminating breakdownmaintenance, and reducing inefficiencies for the users.

The first step is the definition of a database of the annual maintenance operations overall duration for each type of intervention, analyzing the maintenance sheets and integrating data within the DTM.

Therefore, the overall sum of the calculated interventions gives a cost that can be considered as a fixed annual fee for the single user, without variations coming from 
emergency interventions. From a preliminary analysis on a residential district composed of 16 buildings, it emerges how the use of drones integrated with image recognition systems for building monitoring through the same cameras used for security systems, could lead to a decrease in working hours with a consequent annual saving of about $€ 25,000$.

\section{CONCLUSIONS}

The proposed framework shows how the integration of AI in DTM-based management systems can develop cognitive building systems with predictive capabilities, implementing autonomous decisions based on the analysis performed, representing a methodological approach replicable for different areas and uses.

Moreover, the potential of DT systems combined with AI can build an efficient virtual ecosystem of control and management in urban residential areas, as well as for complex infrastructures or machines. In particular, the configuration of a full-digital and integrated system for security management defines an ecosystem of unmanned security potentially integrated with technologies for the optimization of maintenance operations, with the objective of reducing costs and malfunctions, configuring digital systems for predictive maintenance and aiming at the progressive elimination of failures.

Computer vision can address issues associated with human errors in current public space security systems. In fact, an automated security camera system only detects what is programmed to view, reducing errors and ineffectiveness coming from humans' distractions during real-time monitoring. Moreover, AI will not be focused on detecting specific events resulting in important events going unnoticed.

Thus, integrating AI into facility management processes means using software that can autonomously make decisions and solve problems through deduction and reasoning processes by collecting and analyzing large amounts of relevant data, supporting decisions and providing insights [37]. ML elaborates information and provide the ability for intelligent systems to learn automatically and improve themself basing on experience, without being explicitly programmed to do so. Therefore, a major advantage in this regard is the ability to identify and eliminate routine and repetitive tasks [38] that can be easily automated. Therefore, facility management using service automation systems benefits from automating tasks such as recurring scheduled maintenance and mandatory inspections of critical equipment. $\mathrm{AI}$ and $\mathrm{ML}$ then take automation to the advanced level of determining which tasks to automate to achieve increased efficiency, making the system smarter gradually over time as the amount of analyzed data increases.

Further developments on this research will be focused on implementing the proposed framework on an experimental residential compound, determining results in cost savings and automated processes.

\section{REFERENCES}

[1] Nazarenko, A. \& Camarinha-Matos, L.M., Basis for an approach to design collaborative cyber-physical systems. Technological Innovation for Industry and Service Systems, Costa de Caparica, Portugal, 8-10 May 2019, vol. 553, Springer: Cham, Switzerland, pp. 193-205, 2019.

[2] Ruohomäki, T., Airaksinen, E., Huuska, P., Kesäniemi, O. \& Martikka, M. \& Suomisto, J., Smart City Platform Enabling Digital Twin. International Conference on Intelligent Systems (IS), pp. 155-161, 2018. DOI: 10.1109/IS.2018.8710517.

[3] Singapore experiments with its digital twin to improve city life. https://www.smartcitylab.com/blog/digital-transformation/singapore-experimentswith-its-digital-twin-to-improve-city-life/. Accessed on: Jun. 2021. 
[4] Rotterdam's Digital Twin Redefines Our Physical, Digital, and Social Worlds. https://smart-cities-marketplace.ec.europa.eu/news-and-events/news/2019/

rotterdams-digital-twin-redefines-our-physical-digital-social-worlds. Accessed on: Jun. 2021.

[5] International Telecommunication Union, Overview of the Internet of Things, 2012, 14. https://www.itu.int/rec/T-REC-Y.2060-201206-I.

[6] Jara, A.J., Sun, Y., Song, H., Bie, R., Genooud, D. \& Bocchi, Y., Internet of Things for cultural heritage of smart cities and smart regions. 2015 IEEE 29th International Conference on Advanced Information Networking and Applications, IEEE: Gwangiu, pp. 668-675. DOI: 10.1109/WAINA.2015.169, 2015.

[7] Benalla, M., Achchab, B. \& Hrimech, H., A distributed intelligent system for emergency convoy. International Journal of Interactive Multimedia and Artificial Intelligence, 4, p. 42, 2016. DOI: 10.9781/ijimai.2016.418.

[8] Delamo, M., Felici-Castell, S., Pérez-Solano, J.J. \& Foster A., Designing an open source maintenance-free environmental monitoring application for wireless sensor networks. Journal of Systems and Software, 103, pp. 238-247, 2015.

DOI: $10.1016 /$ j.jss.2015.02.013.

[9] González García, C., García-Bustelo, C.P., Espada, J.P. \& Cueva-Fernandez, G., Midgar: Generation of heterogeneous objects interconnecting applications. A domain specific language proposal for internet of things scenarios. Computer Networks, 64, pp. 143-158, 2014. DOI: 10.1016/j.comnet.2014.02.010.

[10] Sanchez, L., et al., SmartSantander: IoT experimentation over a smart city testbed. Computer Networks, 61, pp. 217-238, 2014. DOI: 10.1016/j.bjp.2013.12.020.

[11] McCarthy, O. \& O’Mahony, M., End user response to an event detection and route reconstruction security system prototype for use in airports and public transport hubs. Transportation Research Board 95th Annual Meeting, Washington, DC. http://amonline.trb.org/trb60693-2016-1.2807374/t001-1.2823436/254-1.2823593/ 16-5450-1.2980693/16-5450 1.2993283?qr=1. Accessed on: 1 Jul. 2020.

[12] Mitchell, S., Villa, N., Stewart-Weeks, M. \& Lange, A., The Internet of everything for cities: Connecting people, process, data, and things to improve the "livability" of cities and communities. pp. 1-21, 2013. http://www.cisco.com/web/strategy/docs/gov/ everything-for-cities.pdf.

[13] Ayub Khan, A., Ali Laghari, A. \& Ahmed Awan, S., Machine learning in computer vision: A review. ICST Transactions on Scalable Information Systems, 2021.

[14] Mihai, S. et al., A Digital Twin Framework for Predictive Maintenance in Industry, 4.0, 2021.

[15] Melesse, T.Y., Di Pasquale, V., Riemma, S., Digital twin models in industrial operations: A systematic literature review. Procedia Manufacturing, 2020.

[16] Barricelli, B.R., Casiraghi, E. \& Fogli, D., A survey on digital twin: Definitions, characteristics, applications, and design implications. IEEE Access, 7, pp. 167653167671, 2019. DOI: 10.1109/ACCESS.2019.2953499.

[17] Zheng, Y., Yang, S. \& Cheng, H., An application framework of digital twin and its case study. Journal of Ambient Intelligence and Humanized Computing, 10, pp. 11411153, 2019. DOI: 10.1007/s12652-018-0911-3.

[18] Snijders, R., Pileggi, P., Broekhuijsen, J., Verriet, J., Wiering, M. \& Kok, K., Machine learning for digital twins to predict responsiveness of cyber-physical energy systems. Paper Presented at the 8th Workshop on Modeling and Simulation of Cyber-Physical Energy Systems, MSCPES 2020 - Proceedings, 2020.

DOI: 10.1109/MSCPES49613.2020.9133695. 
[19] Geyer, P. \& Singaravel, S., Component-based machine learning for performance prediction in building design. Applied Energy, 228, pp. 1439-1453, 2018.

DOI: 10.1016/j.apenergy.2018.07.011.

[20] Aivaliotis, P., Georgoulias, K. \& Alexopoulos, K., Using digital twin for maintenance applications in manufacturing: State of the art and gap analysis. Proceedings of the IEEE International Conference on Engineering, Technology and Innovation (ICE/ITMC), IEEE: Piscataway, USA, pp. 1-5, 2019.

[21] Alam, K.M. \& El Saddik, A. C2PS: A digital twin architecture reference model for the cloud-based cyber-physical systems. IEEE Access, 5, pp. 2050-2062, 2017.

DOI: 10.1109/ACCESS.2017.2657006.

[22] Chhetri, S.R., Faezi, S., Canedo, A. \& Faruque, M.A.A., QUILT. Proceedings of the International Conference on Internet of Things Design and Implementation - IoTDI '19, eds. O. Landsiedel \& K. Nahrstedt, Montreal, Canada, ACM Press: New York, USA, pp. 237-248, 2019.

[23] Enders, M.R. \& Hoßbach, N., Dimensions of digital twin applications - A literature review. Proceedings of the 25th Americas Conference on Information Systems, Cancun: Mexico, pp. 1-10, 2019.

[24] Otto, B., Hompel, M. ten \& Wrobel, S. Reference architecture for the digitization of industries. Digital Transformation, ed. R. Neugebauer, Springer Vieweg: Berlin, Germany, pp. 109-128, 2019.

[25] Tao, F. et al., Digital twin-driven product design framework. International Journal of Production Research, 57(12), pp. 3935-3953.

[26] Simplifying 5G with the Network Digital Twin, Spirent. https://www.spirent.com/ assets/wp/wp simplifying-5 g-with-thenetwork-digital-twin. Accessed on: 16 Mar. 2020.

[27] Aivaliotis, P., Georgoulias, K. \& Alexopoulos, K., Using digital twin for maintenance applications in manufacturing: State of the art and gap analysis. IEEE International Conference on Engineering, Technology and Innovation (ICE/ITMC), pp. 1-5, 2019.

[28] Raza, M., Kumar, P.M., Hung, D.V., Davis, W., Nguyen, H. \& Trestian, R., A digital twin framework for industry 4.0 enabling next-Gen manufacturing. 9th International Conference on Industrial Technology and Management (ICITM), Oxford, pp. 73-77, 2020.

[29] Barthelmey, A., Lee, E., Hana, R. \& Deuse, J., Dynamic digital twin for predictive maintenance in flexible production systems. IECON 2019 - 45th Annual Conference of the IEEE Industrial Electronics Society, Lisbon, Portugal, pp. 4209-4214, 2019.

[30] Liang, Y.C., Li, W.D., Lu, X., \& Wang, S., Fog computing and convolutional neural network enabled prognosis for machining process optimization. Journal of Manufacturing Systems, 52, pp. 32-42, 2019.

[31] Xu, L.D., He, W. \& Li, S., Internet of Things in industries: A survey. IEEE Transactions on Industrial Informatics, 10(4), pp. 2233-2243, 2014.

DOI: $10.1109 /$ TII.2014.2300753.

[32] Telikani, A., Gandomi, A.H. \& Shahbahrami, A., A survey of evolutionary computation for association rule mining. Information Sciences, 524, pp. 318-352, 2020. DOI: $10.1016 /$ j.ins.2020.02.073.

[33] Bock, H.H., Clustering methods: A history of k-means algorithms. Selected contributions in data analysis and classification. Studies in Classification, Data Analysis, and Knowledge Organization, eds. P. Brito, G. Cucumel, P. Bertrand \& F. de Carvalho, Springer: Berlin/Heidelberg, Germany, pp. 161-172, 2007.

DOI: 10.1007/978-3-540-73560-1\15. 
[34] Van de Moortel, K., Multidirectional Regression Analysis, 2021. DOI: 10.13140/RG.2.2.16703.64162.

[35] Wang, Y. \& Wang, C., Computer vision-based color image segmentation with improved kernel clustering. International Journal on Smart Sensing and Intelligent Systems, 8, pp. 1706-1729, 2015. DOI: 10.21307/ijssis-2017-826.

[36] Negri, E., Fumagalli, L. \& Macchi, M., A review of the roles of digital twin in CPSbased production systems. Procedia Manufacturing, 11, pp. 939-948, 2017. DOI: 10.1016/j.promfg.2017.07.198.

[37] Napoleone, A., Macchi, M. \& Pozzetti, A., A review on the characteristics of cyberphysical systems for the future smart factories. Journal of Manufacturing Systems, 54, pp. 305-335, 2020. DOI: 10.1016/j.jmsy.2020.01.007.

[38] Marmolejo-Saucedo, J.A., Hurtado-Hernandez, M. \& Suarez-Valdes, R., Digital twins in supply chain management: A brief literature review. International Conference on Intelligent Computing and Optimization, pp. 653-661, 2019. 